\title{
Risk factors for tibial damage associated with the modified Maquet technique in 174 stifles
}

\author{
Michael D. Lefebvre, DVM¹ ～Olivier R. Broux, DVM1 | \\ Nicolas P. Barthélémy, DVM, DECVS² | Martin Hamon, DVM1 | \\ Evelyne V. Moyse, PhD ${ }^{3}$ ｜ Bernard M. Bouvy, DVM, MS, DECVS, Boarded ACVS1 (D) | \\ Marc H. Balligand, DVM, PhD, Cert SAO, DECVS ${ }^{1}$
}

${ }^{1}$ Department of Clinical Sciences - Small Animal Surgery, Faculty of Veterinary Medicine, University of Liège, Liège, Belgium

${ }^{2}$ Langford Veterinary Services, University of Bristol, Langford, North Somerset, United Kingdom

${ }^{3}$ Department of Biostatistics and Bioinformatics applied to Veterinary Sciences, Faculty of Veterinary Medicine, University of Liège, Liège, Belgium

\section{Correspondence}

Michael Lefebvre, Department of Clinical Sciences - Small Animal Surgery, Faculty of Veterinary Medicine, University of Liège, Liège, Belgium. Email: mlefebvre.vet@gmail.com

\begin{abstract}
Objective: To identify risk factors for tibial damage associated with the modified Maquet technique (MMT) in dogs with cranial cruciate ligament (CCL) disease.

Study design: Retrospective study.

Sample population: One hundred and seventy-four stifles from 147 client-owned dogs.

Methods: Medical records of dogs diagnosed with CCL disease and treated with the current version of MMT were reviewed. Dogs were included if immediate postoperative radiographs were available. Cortical hinge fracture or fissure, tibial tuberosity fracture, and diaphyseal fractures of the tibia were recorded. Age, body weight (BW), thickness of the tibial cortical hinge, and angle of opening of the osteotomy were tested as potential risk factors for tibial damage by univariate logistic regression analysis.

Results: Tibial damage included intraoperative tibial fissures in 37\% of MMTs, intraoperative fractures of the cortical hinge in $3.4 \%$ of MMTs, postoperative tibial fractures in $14 \%$ of MMTs. Risk factors for intraoperative fissure included BW $(P=.0153)$ and thickness of cortical hinge $(P=.0006)$. The angle of opening of the osteotomy was identified as a risk factor for intraoperative cortical hinge fracture $(P=.0034)$, angles below $11^{\circ}$ being preventive. No risk factor was identified for postoperative fracture.

Conclusion: Based on these results, preventive measures against tibial damage associated with MMT should include: a thickness of cortical hinge based on the equation related to the BW; a length of osteotomy adjusted to the amount of TTA with an osteotomy angle below $10^{\circ}$; and slow advancement of the tibial tuberosity.
\end{abstract}

\section{1 | INTRODUCTION}

Cranial cruciate ligament (CCL) disease is the most commonly diagnosed pathology in canine orthopedics. ${ }^{1-3}$ Surgery

Results are pending acceptation for the resident forum of the 26th Congress of the European College of the European College of Veterinary Surgery (ECVS), Edinburgh, Scotland, July 13-15, 2017. is considered the best therapeutic regimen. ${ }^{4,5}$ Tibial tuberosity advancement (TTA) and tibial plateau leveling osteotomy are 2 of the most popular techniques currently performed to treat CCL disease. Fracture of the tibial tuberosity is a complication reported after both techniques. ${ }^{6-8}$ Several risk factors have been identified for this complication, such as shape of the osteotomy, position of the plate and cage, body weight 


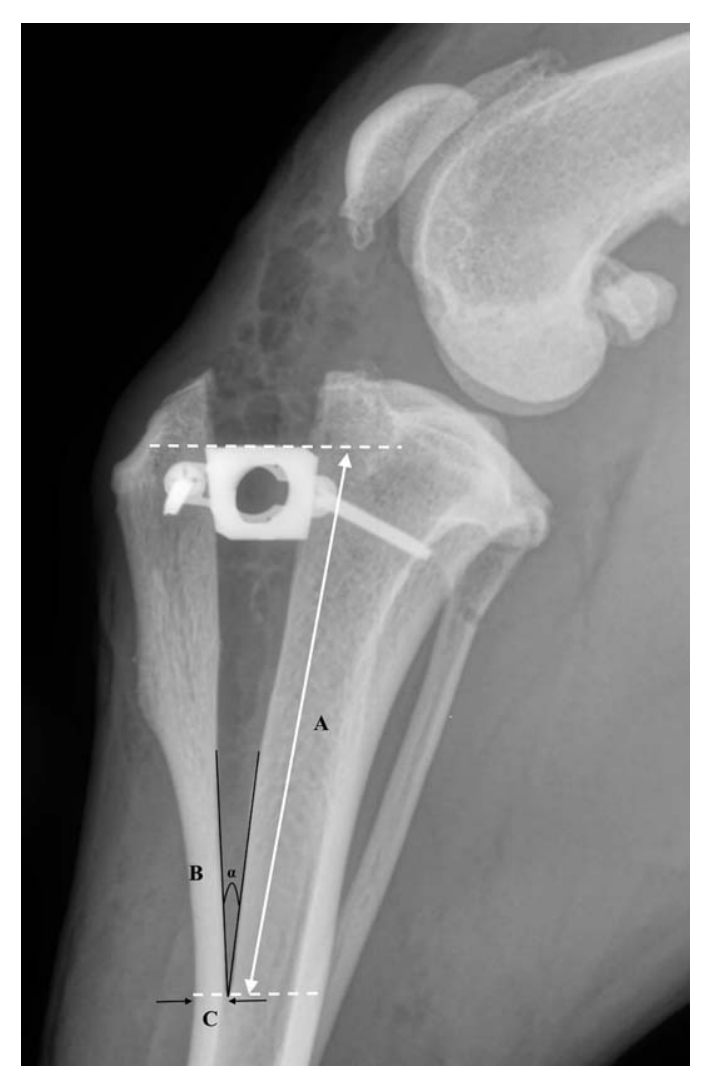

FIGURE 1 Illustration of a MMT performed in the present study. Long osteotomy (up to around $2 \times$ length of tibial crest) is performed. The desired cranio-caudal thickness of the cortical hinge is determined using the following formula: Thickness $(\mathrm{mm})=(6 \times \mathrm{BW}(\mathrm{N})+417.561-$ $15.68 \times \mathrm{BW}(\mathrm{kg})) / 508$. A (between dotted lines): length of osteotomy; B (black line): angle of opening $(\alpha)$; $\mathrm{C}$ (between black arrows): cortical hinge

(BW), preoperative patellar tendon angle, and width of the tibial tuberosity. ${ }^{9-11}$

In 2011, the modified Maquet technique (MMT) was introduced as an alternative option for TTA. ${ }^{12}$ The technique preserved the distal cortical hinge (remaining bony attachment) of the tibial crest, hence avoiding plate fixation of the tibial tuberosity. The MMT has been refined over time, from a rather short osteotomy $(1 \times$ length of tibial crest) with a hole drilled at its distal extremity to, currently, a much longer osteotomy (up to around $2 \times$ length of tibial crest) with no hole. ${ }^{13,14}$ These changes were mainly aimed at facilitating the cranial displacement of the tibial tuberosity, while reducing the risk of fissure or fracture of the distal cortical hinge, sometimes observed with the previous version of the MMT. However, the influence of these modifications on the incidence of tibial damage has not been studied. This study was, therefore, designed to review the incidence of cortical hinge fracture/fissure, tibial tuberosity fracture, and diaphyseal fractures of the tibia. The second objective was to identify risk factors for tibial damage associated with the MMT, as a basis for current recommendations.

\section{2 | MATERIALS AND METHODS}

Medical records of dogs diagnosed with partial or total CCL rupture between September 2011 and August 2016 at the the Clinique Vétérinaire Universitaire (University of Liege) were reviewed. Inclusion criteria included CCL disease treated with the latest version of MMT (Figure 1) and availability of at least an immediate postoperative radiograph. MMT was performed with the elongated osteotomy design recently reported (1.5-2× length of tibial crest) ${ }^{13}$ created with an oscillating saw under irrigation. No hole was drilled at the distal aspect of the osteotomy. The cranio-caudal thickness of the cortical hinge was preoperatively determined according to the formula previously published ${ }^{13}$ : Thickness $(\mathrm{mm})$ $=\frac{6 \times \mathrm{BW}(\mathrm{N})+417.561-15.68 \times \mathrm{BW}(\mathrm{kg})}{508}($ Table 4). A titanium cage of appropriate size was fixed with 2 self-taping titanium cortical screws (Kyon TTA-1, Zurich, Switzerland) to maintain the advancement. The proximal aspect of the cage was located at the level of insertion of the patellar ligament. Two diplomates of the European College Veterinary Surgeons (ECVS) and 7 ECVS residents performed the procedures.

Data recorded for subsequent analysis included age, BW, breed, gender, time between surgery and radiographic recheck,

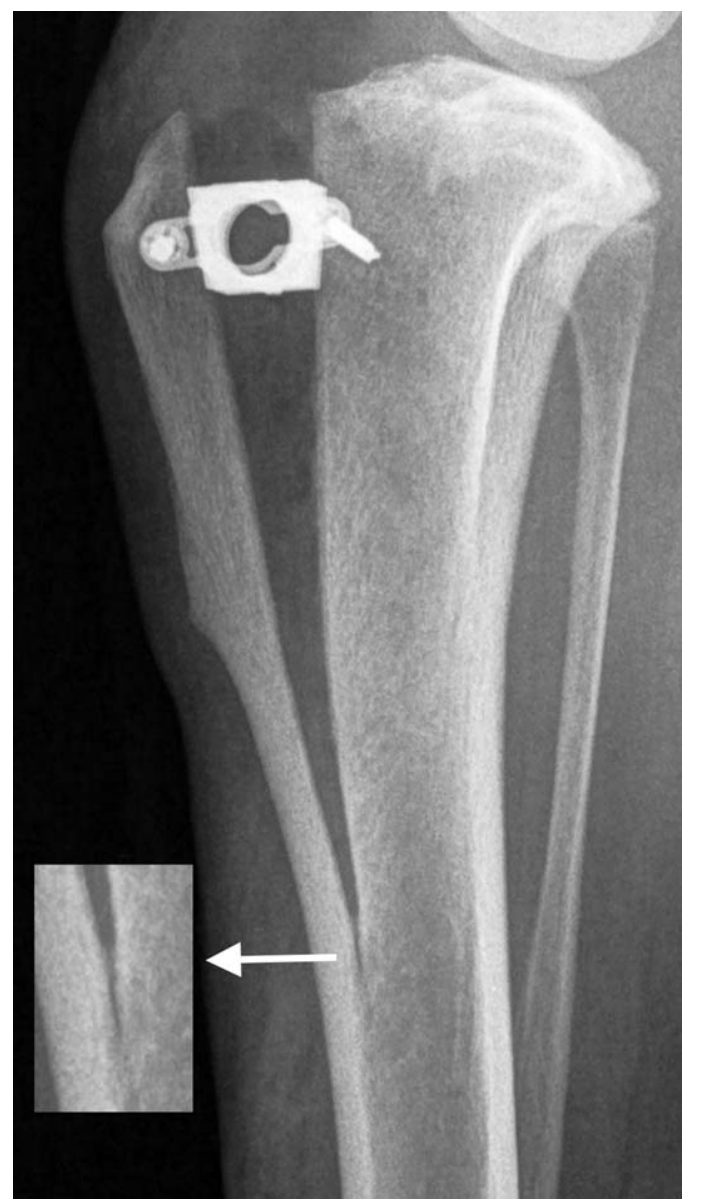

FIG URE 2 Illustration of an intraoperative fissure following MMT (white arrow) 
other stifle operated, left or right stifle, size of cage, thickness of the tibial cortical hinge, and angle of opening of the osteotomy.

Cortical hinge fracture or fissure, tibial tuberosity fracture, and diaphyseal fractures of the tibia were recorded. Intraoperative cortical hinge fissure was defined as a distal extension of the osteotomy line on postoperative radiographs (Figure 2). Cortical hinge fracture was defined as a complete separation between the tibial shaft and the tibial crest observed during surgery or on immediate postoperative radiographs.

\section{1 | Statistical analysis}

Statistical analysis was performed using statistical software programs (SAS 9.3, SAS Institute, Tervueren, Belgium, and XLstats, Addinsoft, Paris, France). Descriptive statistics included age, BW, breed, and gender. Continuous data were reported as median and range. Univariate logistic regression analysis was used to assess the association between the occurrence of tibial damage and the suggested risk factors. The $P$-value and odds ratio (OR) with $95 \%$ confidence interval (CI) were recorded for each variable. Results were considered to be significant at $P$-value $<.05$.

\section{3 | RESULTS}

\section{1 | Study population}

Medical records from 147 client-owned dogs fulfilled the inclusion criteria. Twenty-seven (18.4\%) dogs had staged bilateral MMTs, leading to 174 MMTs performed on 88 right and 86 left stifles. The median age and BW of dogs were 6.0 years (10 months to 14 years) and $28.8 \mathrm{~kg}(1.8-70.0 \mathrm{~kg})$, respectively. This population included 89 female and 58 male dogs, consisting of 27 mixed breeds, 10 Labrador Retrievers, 9 American Staffordshire Terriers, 8 Golden Retrievers, 6 Boxers, 6 Bernese Mountain Dogs, 6 German Shepherds, 6 Jack Russell Terriers, 6 Cane Corsos, and 40 other breeds with 1 to 5 individuals per breed. Cage sizes were $3,4.5,6,9,10.5$, 12 , and $15 \mathrm{~mm}$ in 5, 15, 21, 32, 1, 67, and 33 MMTs, respectively. The median thickness of the cortical hinge and angle of opening of the osteotomy measured on immediate postoperative radiographs were $3.8 \mathrm{~mm}(1.0-10.3 \mathrm{~mm})$ and $9.0^{\circ}\left(4.4^{\circ}\right.$ $19.0^{\circ}$ ), respectively. Radiographic re-evaluation was available in 148 MMTs, at 47 days (4 days to 2.1 years), while 15\% (26/ 174) of cases did not return for radiographic recheck.

\subsection{Assessment of tibial damage}

Intraoperative fissures were detected on immediate postoperative radiograph in 37\% (64/174) of MMTs. Among these, 3 were treated with 1 lag screw, 4 with 1 Kirschner wire, and 1 with 1

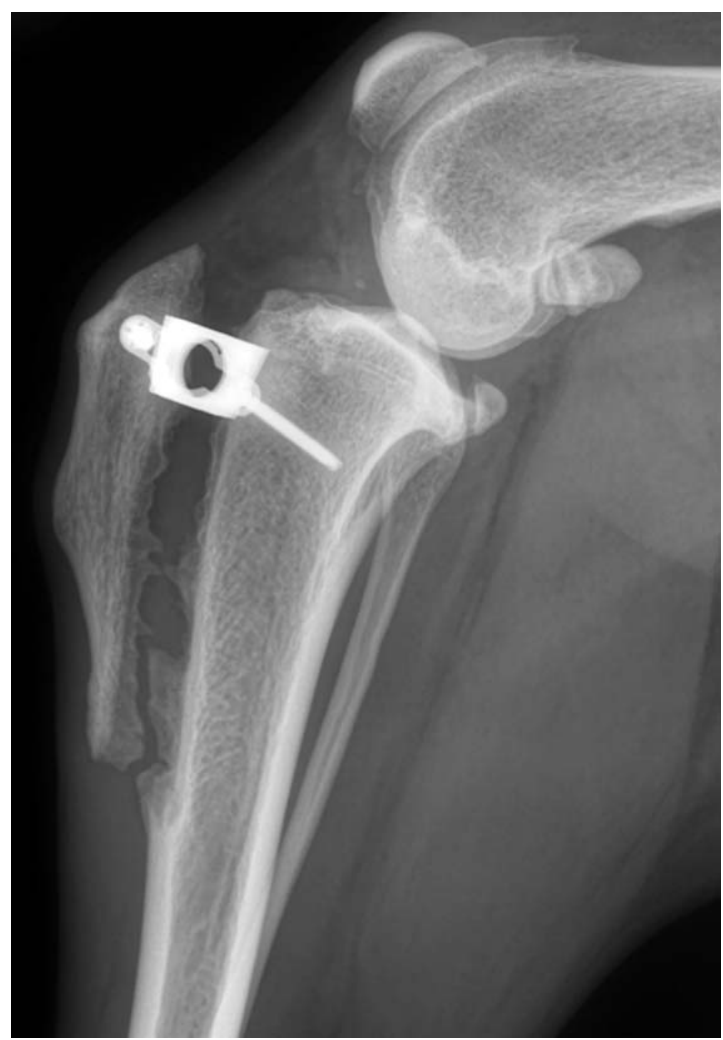

F IG URE 3 Illustration of a cortical hinge fracture, 47 days post-op. Bone healing is progressing

Kirschner wire and a figure-of-eight cerclage wire applied as a tension-band wire. The remaining 56 fissures were left untreated.

Intraoperative fractures of the cortical hinge were diagnosed in 3.4\% (6/174) of MMTs. All were treated, 4 with 1 figure-of-eight cerclage wire, 1 with 1 Kirschner wire, and 1 with 2 Kirschner wires.

Postoperative fractures of the tibia were noted on radiographic re-evaluation in 14.2\% (21/148) of MMTs. Fracture of the cortical hinge (Figure 3), tibial tuberosity (Figure 4), and tibial shaft (Figure 5A,B) occurred in 17, 2, and 2 MMTs, respectively. These complications were diagnosed at a median follow-up time of 46 days ( 4 days to 2.1 years). Tibial shaft fractures were diagnosed at 4 and 14 days. Tibial tuberosity fractures were diagnosed at 35 and 773 days. About 29\% (6/ 21 ) of the postoperative fractures ( 1 diaphyseal and 5 cortical hinge fractures) were subsequent to an intraoperative fissure. The cortical hinge and tibial tuberosity fractures were treated conservatively based on their limited displacement and progression of bone healing at the time of diagnosis (Figures 3 and 4). Both tibial shaft fractures were treated with plate osteosynthesis. All fractures healed uneventfully.

\section{3 | Risk factors for tibial damage}

The angle of opening of the osteotomy (OR: 1.670, CI 95\%: $1.185-2.353, P=.0034$ ) influenced the occurrence of perioperative fractures of the cortical hinge (Table 1). The 


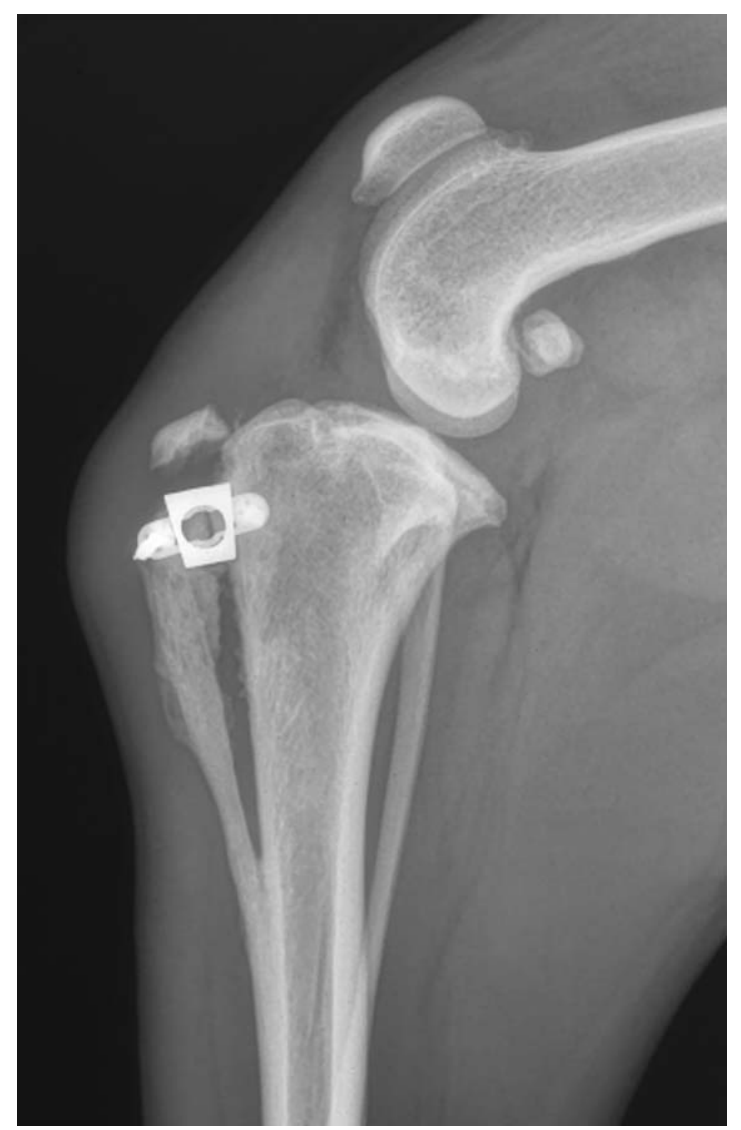

F IG URE 4 Illustration of a tibial tuberosity fracture, 35 days post-op

likelihood of fracture dropped below 5\% with angles below $11^{\circ}$. The thickness of the cortical hinge was associated (OR: 1.607, CI 95\%: 1.226-2.107, $P=.0006)$ with intraoperative fissures of the cortical hinge. Similarly, increasing BW (OR: 1.028, CI 95\%: 1.005-1.052, $P=.0153$ ) was associated with intraoperative fissures of the cortical hinge.

Tibial shaft fractures occurred in only 2/174 MMTs, one of these in a heavy dog $(70 \mathrm{~kg})$. Technical mistakes occurred in both cases in one case a pin placed to stabilize a fractured

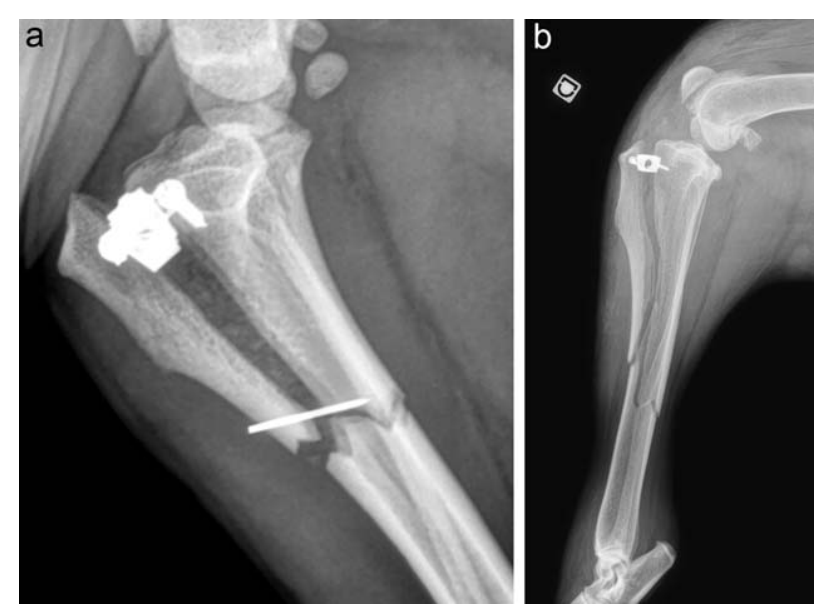

F IG URE 5 A, Illustration of a tibial shaft fracture, 14 days post-op. $\mathrm{B}$, Illustration of a tibial shaft fracture, 4 days post-op ${ }^{14}$
TA B LE 1 Results of univariate logistic regression analysis ( $P$-values in bold characters are significant)

\begin{tabular}{|c|c|c|c|}
\hline Risk factors & $\begin{array}{l}\text { Intra-op. } \\
\text { fissure }\end{array}$ & $\begin{array}{l}\text { Intra-op. } \\
\text { fracture }\end{array}$ & $\begin{array}{l}\text { Post-op. } \\
\text { fracture }\end{array}$ \\
\hline Age & 0.7270 & 0.8724 & 0.1035 \\
\hline Body weight & 0.0153 & 0.2514 & 0.9690 \\
\hline Angle of opening & 0.9950 & 0.0034 & 0.8317 \\
\hline $\begin{array}{l}\text { Thickness of } \\
\text { cortical hinge }\end{array}$ & 0.0006 & 0.6637 & 0.0559 \\
\hline
\end{tabular}

cortical hinge had weakened the tibial shaft (Figure 5A) and in the other case, multiple erratic osteotomy planes had been created (Figure 5B). No risk factors were detected for postoperative fractures of the cortical hinge.

\section{4 | DISCUSSION}

This study identified the angle of opening of the osteotomy line and the thickness of the cortical hinge as the main factors increasing the risk of peri-operative tibial damage during MMT in dogs.

Intraoperative fissures occurred more frequently than intraoperative fractures, and were located most commonly at the distal aspect of the osteotomy line. Thickness of the cortical hinge $(P=.0006)$ and $\mathrm{BW}(P=.0153)$ were the sole risk factors associated with intraoperative fissures. The thickness of the cortical hinge is preoperatively determined using a previously published formula. ${ }^{13}$ The thickness of the hinge increased with the BW of the dog, in order to resist the tensile stress applied by the quadriceps muscle during weight bearing. Greater thickness of the hinge increases its rigidity, affecting its capacity to deform under stress. A thicker cortical hinge is therefore predisposed to longitudinal fissures during advancement of the tibial tuberosity. These fissures are parallel to the cranial tibial cortex, mimicking a distal extension of the osteotomy line, allowing the tibial tuberosity advancement with a lesser deformation of the hinge. Although intraoperative fissures were detected in 37\% (64/174) of MMTs, only 9.4\% (6/ 64) subsequently led to postoperative tibial fractures, which did not appear to be a great concern. Intraoperative fractures were associated with the angle of opening of the osteotomy line $(P=.0034)$. These intraoperative fractures are caused by the stress applied to the cortical hinge during tibial tuberosity advancement. Angles equal to or below $10^{\circ}$ of opening of the osteotomy line are associated with a low risk of fractures $(P<.030)$, while the risk of fracture increases considerably and becomes significant above $11^{\circ}$ of opening $(P>.053)$ (Table 2). A preventive strategy therefore consists of increasing the length of the osteotomy with the desired amount of TTA, applying the trigonometric equation: length of osteotomy $(\mathrm{mm})=\frac{\text { TTA }(\mathrm{mm})}{\tan (\alpha)}$, where $\alpha$ is the angle 
TABLE 2 Influence of the angle of opening of the osteotomy on the probability of intraoperative fractures

\begin{tabular}{|ll|}
$\begin{array}{l}\text { Angle of } \\
\text { opening }\left({ }^{\circ}\right)\end{array}$ & $\begin{array}{l}\text { Probability of } \\
\text { fracture }(\%)\end{array}$ \\
\hline 5 & 0.16 \\
\hline 6 & 0.29 \\
\hline 7 & 0.53 \\
\hline 8 & 0.95 \\
\hline 9 & 1.70 \\
\hline 10 & 3.03 \\
\hline 11 & 5.33 \\
\hline 12 & 9.21 \\
\hline 13 & 15.45 \\
\hline 14 & 24.77 \\
\hline 15 & 37.22 \\
\hline
\end{tabular}

of opening $\leq 10^{\circ}$ (Figure 6). Considering that TTA ordinarily ranges from 3 to $15 \mathrm{~mm}$, the length of the osteotomy should comprise between $150 \%$ and $200 \%$ of the length of the tibial crest, possibly even a little longer. Application of the trigonometric formula for $\alpha$ fixed at $<10^{\circ}\left(9^{\circ}\right.$ or $\left.8^{\circ}\right)$ allows

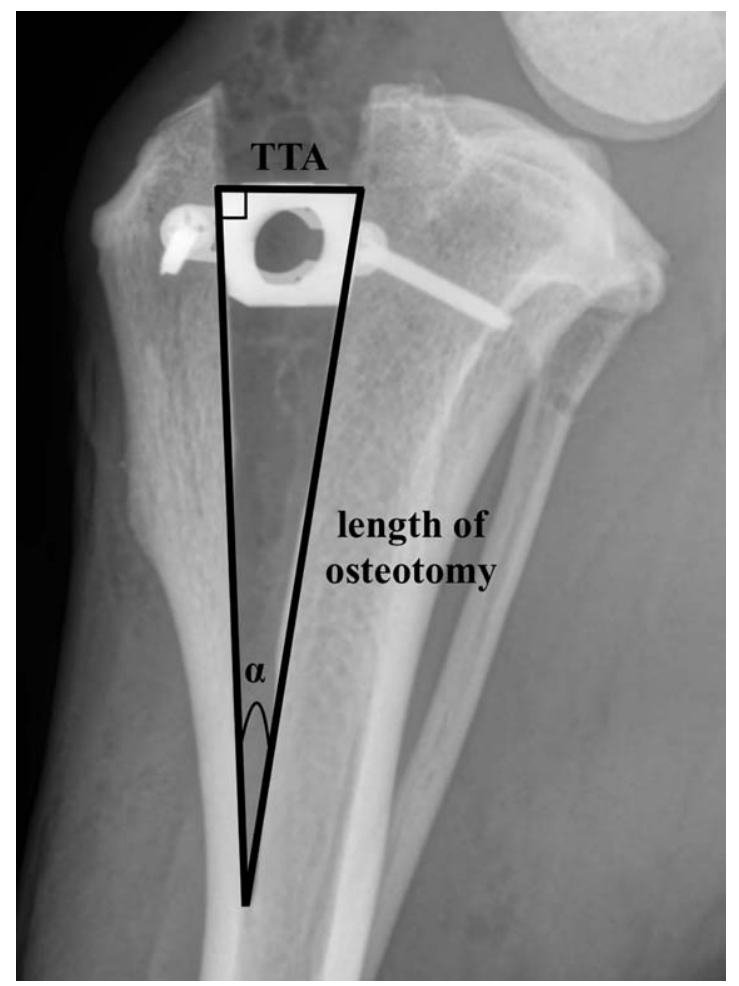

F IGURE 6 Illustration of trigonometric formula used to determine length of osteotomy according to tibial tuberosity advancement (TTA) in order to have an angle of opening $(\alpha)<$ or equal to $10^{\circ} \cdot \tan (\alpha)=$ (TTA $(\mathrm{mm})) /($ length of osteotomy $(\mathrm{mm})$ )
TA B LE 3 Length of the osteotomy derived from the trigonometric formula: length of osteotomy $(\mathrm{mm})=\frac{\text { TTA }(\mathrm{mm})}{\tan (\alpha)}$, with $\quad$ TTA $=$ tibial tuberosity advancement and $\alpha=$ angle of opening of the osteotomy

\begin{tabular}{|c|c|c|}
\hline $\begin{array}{l}\text { Cage } \\
(\mathrm{mm})\end{array}$ & $\begin{array}{l}\text { Length of } \\
\text { osteotomy (mm) } \\
\text { for } \alpha=9^{\circ}\end{array}$ & $\begin{array}{l}\text { Length of } \\
\text { osteotomy ( } \mathrm{mm} \text { ) } \\
\text { for } \alpha=8^{\circ}\end{array}$ \\
\hline 3 & 18.9 & 21.3 \\
\hline 4.5 & 28.4 & 32.0 \\
\hline 6 & 37.9 & 42.7 \\
\hline 9 & 56.8 & 64.0 \\
\hline 12 & 75.8 & 85.4 \\
\hline 15 & 94.7 & 106.7 \\
\hline
\end{tabular}

determination of the length of the osteotomy (Table 3). Extending the osteotomy further distally would decrease the angle of opening even more, but is not justified based on the very low incidence of fracture with angles below $10^{\circ}$.

The speed at which the TTA is carried out was not evaluated in this study but could influence the development of fissures and fractures. Cortical bone is a visco-elasto-plastic material, with a modulus of elasticity increasing with the strain rate. ${ }^{15}$ The cortical hinge should tolerate less deformation when advanced quickly, likely increasing the risk of fissure or fracture, particularly if the opening angle of the osteotomy reaches or exceeds $10^{\circ}$. Gradual advancement of the tibial tuberosity over several minutes, using $3 \mathrm{~mm}$ increments (corresponding to the increment between Kyon spacers), should allow the bone to adapt to the deformation after each stage. This mechanism could explain the lower incidence of fissure or fracture clinically observed by the authors.

Finally, postoperative fractures of cortical hinge occurred in 11\% (17/148) of MMTs with radiographic re-evaluation. Surprisingly, the thickness of the cortical hinge does not appear to influence these complications $(P=.0559)$. This apparent lack of association may reflect the method used by the authors to determine thickness of the cortical hinge, according to the equation based on $\mathrm{BW}$ and published by Brunel et al. ${ }^{13}$ These authors later advised to use $6 \times \mathrm{BW}$ in the formula in order to increase the safety margin until bone healing (Table 4). Increasing the thickness beyond this would decrease the risk of postoperative fracture, but would concomitantly increase the rigidity of the hinge, complicating TTA and increasing the risk of intraoperative fissure or fracture. Following the guidelines provided by the formula does not eliminate postoperative fractures. Other factors may account for these, such as high levels of postoperative activity generating high tensile forces on the tibial tuberosity. Another cause of failure could relate to the mineral composition of the cortical bone where bone density, and hence bone 
TABLE 4 Thickness of the cortical hinge derived from the body weight $(\mathrm{BW})$ and calculated with the formula published by Brunel et $\mathrm{al}^{13}$

\begin{tabular}{|llll|}
\hline BW $(\mathbf{k g})$ & Thickness $(\mathbf{m m})$ & BW $(\mathbf{k g})$ & Thickness $(\mathbf{m m})$ \\
\hline 3 & 1.08 & 30 & 3.37 \\
\hline 6 & 1.33 & 40 & 4.22 \\
\hline 10 & 1.67 & 50 & 5.07 \\
\hline 15 & 2.10 & 60 & 5.92 \\
\hline 20 & 2.52 & 70 & 6.77 \\
\hline 25 & 2.95 & 80 & 7.62 \\
\hline
\end{tabular}

mechanical performance, may be decreased in patients with osteoporosis. Finally, technical errors and lack of accuracy could lead to a thinner hinge than planned, thereby increasing the risk of traumatic postoperative fracture. Tibial shaft fractures occurred in only 2/174 MMTs, both with technical mistakes. Extending the osteotomy of the tibial crest within the tibial diaphysis opens its otherwise closed cylindrical morphology, thereby affecting its biomechanical properties. Yet, the tibia generally seems to cope with the postoperative stresses until bone healing is complete. The tibial crest, cage, and tibial shaft form a construct seam sharing the load of forces applied on the tibia, avoiding excessive stress concentration on the tibial shaft, caudal to the osteotomy.

Limitations of this study are inherent to its retrospective nature and include inconsistent follow-up, as 26 cases did not come back for radiographic re-evaluation. This limitation may have affected the identification of risk factors for postoperative fractures. The technique has continued to evolve based on the authors' clinical experience, and additional factors may be influencing outcomes. Based on the results of this study and the authors' experience (6 years), the following recommendations can be made in order to minimize the risk of tibial damage during MMT: (1) the thickness of the cortical hinge should be calculated according to $\mathrm{BW}$ of the patient via the formula published by Brunel et $\mathrm{al}^{13}$ and (2) the length of the osteotomy should be calculated according to the width of the cage in order to keep the opening angle of the tibial tuberosity equal to or below $10^{\circ}$. Although not tested here, the viscoelastic properties of bone provide a basis to recommend a slow and gradual advancement of the tibial tuberosity, in $3 \mathrm{~mm}$ increments.

\section{CONFLICT OF INTEREST}

The authors declare no conflict of interest related to this report.

\section{ORCID}

Bernard M. Bouvy (1) http://orcid.org/0000-0002-9465-8406

\section{REFERENCES}

[1] Kowaleski MP, Boudrieau RJ, Pozzi A. Stifle joint. In: Tobias KM, Johnston SA, eds. Veterinary Surgery Small Animal. Vol 1. St. Louis, MO: Elsevier; 2013:906-998.

[2] Johnson JA, Austin C, Breur GJ. Incidence of canine appendicular musculoskeletal disorders in 16 veterinary teaching hospitals from 1980 through 1989. Vet Comp Orthop Traumatol. 1994;7:56-69.

[3] Whitehair JG, Vasseur PB, Willits NH. Epidemiology of cranial cruciate ligament rupture in dogs. J Am Vet Med Assoc. 1993;203:1016-1019.

[4] Mölsä SH, Hyytiäinen HK, Hielm-Björkman AK, Laitinen-Vapaavuori OM. Long-term functional outcome after surgical repair of cranial cruciate ligament disease in dogs. BMC Vet Res. 2014;10:266.

[5] Wucherer KL, Conzemius MG, Evans R, Wilke VL. Short-term and long-term outcomes for overweight dogs with cranial cruciate ligament rupture treated surgically or nonsurgically. $J$ Am Vet Med Assoc. 2013;242:1364-1372.

[6] Calvo I, Aisa J, Chase D, Garcia-Fernandez P, San Roman F, Bennett D. Tibial tuberosity fracture as a complication of tibial tuberosity advancement. Vet Comp Orthop Traumatol. 2014;2:148-154.

[7] de Lima Dantas B, Sul R, Parkin T, Calvo L. Incidence of complications associated with tibial tuberosity advancement in Boxer dogs. Vet Comp Orthop Traumatol 2016;1:39-45.

[8] Stauffer KD, Tuttle TA, Elkins AD, Wehrenberg AP, Character BJ. Complications associated with 696 tibial plateau leveling osteotomies (2001-2003). J Am Anim Hosp Assoc. 2006;1:44-50.

[9] Nutt AE, Garcia-Fernandez P, San Roman F, Parkin T, Calvo I. Risk factors for tibial tuberosity fracture after tibial tuberosity advancement in dogs. Vet Comp Orthop Traumatol. 2015;28:116-123.

[10] Bergh MS, Rajala-Schultz P, Johnson KA. Risk factors for tibial tuberosity fracture after tibial plateau leveling osteotomy in dogs. Vet Surg. 2008;37:374-382.

[11] Wolf RE, Scavelli TD, Hoelzler MG, Fulcher RP, Bastian RP. Surgical and postoperative complications associated with tibial tuberosity advancement for cranial cruciate ligament rupture in dogs: 458 cases (2007-2009). J Am Vet Med Assoc. 2012;12:1481-1487.

[12] Etchepareborde S, Brunel L, Bollen G, Balligand M. Preliminary experience of a modified Maquet technique for repair of cranial cruciate ligament rupture in dogs. Vet Comp Orthop Traumatol. 2011;3:223-227.

[13] Brunel L, Etchepareborde S, Barthélémy N, Farnir F, Balligand M. Mechanical testing of a new osteotomy design for tibial tuberosity advancement using the modified Maquet technique. Vet Comp Orthop Traumatol. 2013;1:47-53.

[14] Ramirez JM, Barthélémy N, Noël S, et al. Complications and outcome of a new modified Maquet technique for treatment of cranial cruciate ligament rupture in 82 dogs. Vet Comp Orthop Traumatol. 2015;5:339-346.

[15] Lakes R. Viscoelastic properties of cortical bone. In: Cowin SC, ed. Bone Mechanics Handbook. 2nd ed. Boca Raton, FL: CRC Press; 2001:11.

How to cite this article: Lefebvre MD, Broux OR, Barthélémy NP, et al. Risk factors for tibial damage associated with the modified Maquet technique in 174 stifles. Veterinary Surgery. 2018;47:30-35. https://doi. org/10.1111/vsu. 12707 